\title{
Transfer of Visuomotor Adaptation to Unpractised Hands and Sensory Modalities
}

\author{
Otmar Bock ${ }^{1}$, Gerd Schmitz ${ }^{2}$ \\ ${ }^{1}$ Institute of Physiology and Anatomy, German Sport University Cologne, Cologne, Germany \\ ${ }^{2}$ Institute of Sports Science, Leibniz University Hannover, Hannover, Germany \\ Email: bock@dshs-koeln.de
}

Received September $18^{\text {th }}, 2013$; revised October 23 $3^{\text {rd }}, 2013$; accepted November $21^{\text {st }}, 2013$

\begin{abstract}
Copyright (C) 2013 Otmar Bock, Gerd Schmitz. This is an open access article distributed under the Creative Commons Attribution License, which permits unrestricted use, distribution, and reproduction in any medium, provided the original work is properly cited. In accordance of the Creative Commons Attribution License all Copyrights (C) 2013 are reserved for SCIRP and the owner of the intellectual property Otmar Bock, Gerd Schmitz. All Copyright (C) 2013 are guarded by law and by SCIRP as a guardian.
\end{abstract}

A recent model (Bock, 2013) predicts that sensorimotor adaptation, achieved while pointing at visual targets, will transfer fully to acoustic targets. The model further predicts that visual-to-acoustic transfer is not diminished even if the left and right arms have adapted to a different distortion. To scrutinize these predictions, we asked subjects to point at visual targets with their right hands under a +30 deg rotation of visual feedback (group "single"), or alternately, with their right hands under a +30 deg and with their left hands under a -30 deg rotation of visual feedback. Aftereffects were registered for each hand and for visual as well as acoustic targets, in counterbalanced order. We found that acoustic aftereffects were only about $66 \%$ of visual ones, which violates the first prediction and calls for an amendment of the model. We further found that acoustic aftereffects were of similar magnitude in both groups, which supports the second prediction. Finally, we observed an intermanual transfer of only about $29 \%$. These findings suggest that unpractised acoustic inputs are weighted somewhat lower than practised visual ones, and that outputs to the unpractised left hand are weighted substantially lower than those to the practised right hand.

Keywords: Sensorimotor Adaptation; Motor Learning; Intermanual; Intersensory; Transfer

\section{Introduction}

Sensorimotor adaptation is not limited to the task by which it was established, but rather can transfer to unpractised motor systems (Cohen, 1973; Cotti, Guillaume, Alahyane, Pélisson, \& Vercher, 2007), new target locations (Bock, 1992; Malfait, Gribble, \& Ostry, 2005) and different distortions (Thomas \& Bock, 2010). These findings should not be taken as evidence that adaptation is achieved by one common process, shared by all motor systems, target locations and distortions. Rather, multiple adaptive processes seem to exist since subjects can concurrently adapt to two distinct distortions, administered in dependence on the arm used (Prablanc, Tzavaras, \& Jeannerod, 1975; Thomas \& Bock, 2012), on target location (Alahyane, 2004; Woolley, Tresilian, Carson, \& Rick, 2007) or on contextual cues (Wada et al., 2003). We are thus left with the puzzling fact that adaptation can transfer from one arm to the other-as if both arms shared a common adaptive process - whilst both arms also can adapt differently — as if each had its own adaptive process.

A model has recently been proposed to explain this apparent discrepancy. As shown in Figure 1, it stipulates that multiple sensory modalities serve as inputs to multiple adaptive mechanisms which, in turn, send their outputs to multiple motor systems via a context-dependent switch; the outputs are differently weighted for practised and for unpractised motor systems. According to this model, single-limb practice doesn't affect the switch position and thus yields transfer of adaptation, while two-limb practice changes the switch position in dependence on the currently active motor system and thus yields limb-specific adaptation.

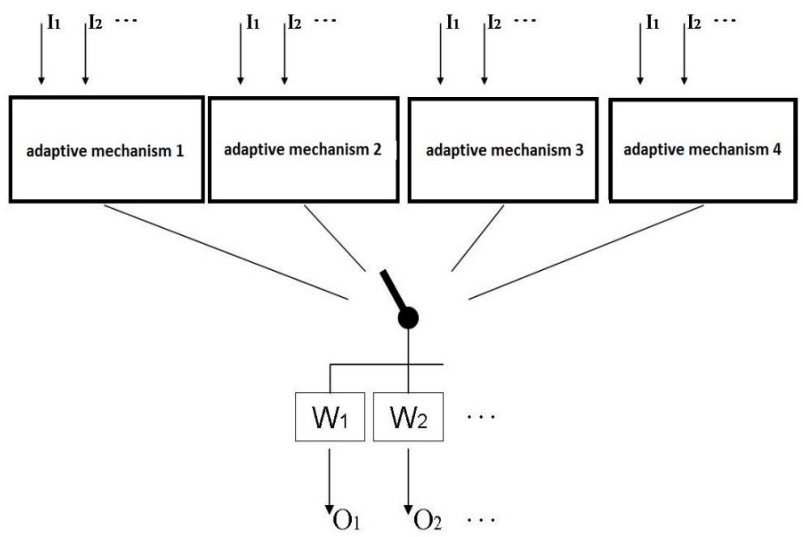

Figure 1.

Conceptual model of sensorimotor adaptation. Sensory inputs from different modalities $\left(\mathrm{I}_{1}, \mathrm{I}_{2}, \ldots\right)$ are transformed by multiple adaptive mechanisms, and their outputs are connected by a context-dependent switch to various motor outputs $\left(\mathrm{O}_{1}, \mathrm{O}_{2}, \ldots\right)$. The connections are weighted differently $\left(\mathrm{W}_{1}, \mathrm{~W}_{2}, \ldots\right)$ depending on whether the motor system is practised or not. Reprinted with modifications from (Bock, 2013). 
The present study scrutinizes two predictions of the published model. First, practised and unpractised motor systems are assigned by different weights, while practised and unpractised sensory modalities are not; if so, adaptation should transfer partially between arms but fully between targets from different modalities. It has indeed been shown that adaptation with visual targets transfers to acoustic targets (Zwiers, van Opstal, \& Paige, 2003) and that aftereffects are equal in both modalities (Kagerer \& Contreras-Vidal, 2009); however the latter study always tested acoustic targets first and visual targets thereafter, so that a possible visual advantage might have dissipated by the time of testing. To overcome this problem, we now test both arms and modalities in counterbalanced order.

According to the second prediction of the published model, both arms can concurrently adapt to different distortions without interference, and this dual adaptation should again transfer fully between sensory modalities. The first part of this predicttion has been confirmed in literature (Bock, Worringham, \& Thomas, 2005; Prablanc et al., 1975), and we now address the second part.

\section{Methods}

25 male and 23 female subjects aged $22.0 \pm 1.6$ years participated after signing their informed consent to this study, which was approved in advance by the first author's Ethics Committee. Subjects were right-handed, healthy, and had no prior experience with adaptation research. As shown by the inset of Figure 1, subjects faced an opaque horizontal panel with a wooden dowel protruding downwards underneath their chin. Targets were presented on the upper surface of the panel, along a semicircle of $36 \mathrm{~cm}$ radius around the dowel. They were presented in balanced order at $\pm 30, \pm 18$, and \pm 6 deg about straight-ahead, either as light dots of $1.5 \mathrm{~cm}$ radius, or as sounds (mix of 0.45 , $1.35,2.30$ and $3.20 \mathrm{kHz}$ ) from loudspeakers of $1.5 \mathrm{~cm}$ radius; the loudspeaker array was hidden from view by a fabric screen. Subjects pointed with their index fingertip underneath the panel, moving from the dowel towards each target where the radial response component was stopped by a semicircular barrier. Subjects then moved the finger along that barrier until it was aligned with the target. They couldn't see their arm and hand because of the opaque panel, but index fingertip position was registered by the Fastrak ${ }^{\circledR}$ system (resolution $1 \mathrm{~mm}$ and $17 \mathrm{~ms}$ ), and could be displayed as a cursor on the upper surface of the panel to provide real-time visual feedback.

The experiment was subdivided into pointing episodes of 45 s duration (or about 25 responses), separated by rest breaks of 5 s. Each experiment started with six baseline episodes. In the first two, subjects pointed with their right hand at visual targets and received veridical visual feedback. In the remaining four baseline episodes, they pointed without visual feedback at visual targets with their right hand (VR), at visual targets with their left hand (VL), at acoustic targets with their right hand (AR), and at acoustic targets with their left hand (AL); the order of these episodes was counterbalanced across subjects. In the subsequent adaptation episodes, subjects pointed at visual targets under rotated visual feedback. One half of them formed group "single", which used their right hand under a +30 deg rotated feedback. The other half formed group "dual", which alternately used their right hand under a +30 deg rotated feedback, and their left hand under a -30 deg rotated feedback. Group "single" performed 20 and group "dual" 40 adaptation episodes, i.e., there were 20 adaptation episodes per hand in both groups. The experiment concluded with four aftereffect episodes, which replicated VR, VL, AR and AL, again in counterbalanced order.

The registered finger position data were analysed by an interactive computer routine which determined the directional error of each response, defined as angle between target and cursor direction $166 \mathrm{~ms}$ after movement onset. This quantifies the feedforward component of motor control without confounding it with feedback-based error corrections, which emerge later during the response. For graphical presentation and statistics, we calculated the mean error of each adaptation episode minus that of the second baseline episode, and the mean errors of each aftereffect episode minus that of the pertinent baseline episode. To facilitate comparisons, data yielded with the left hand of group "dual" were sign-reversed. The outcome was submitted to analyses of variance (ANOVAs) with Greenhouse-Geyser corrections if variances were unequal.

\section{Results}

Figure 2 illustrates the errors of group "single" and "dual" throughout the adaptation block. For clarity, the left hand of group "dual" is plotted in same episodes as the right hand although the data actually came from separate, interleaved episodes. ANOVA with the between-factor Task (single, dual right, dual left) and the within-factor Episode $(1,2, \ldots, 20)$, yielded significance only for Episode $\left(\mathrm{F}_{(19,1292)}=13.15, p<0.001\right)$.

The aftereffects of both groups are summarized in Figure 3 . ANOVA with the between-factor Group (single, dual) and the within-factors Hand $(\mathrm{R}, \mathrm{L})$ and Modality $(\mathrm{V}, \mathrm{A})$ yielded significance for Group $(\mathrm{F}(1,46)=5.83 ; p<0.05)$, Hand $(\mathrm{F}(1,46)=$ $8.46 ; p<0.01)$ and Group*Hand $(\mathrm{F}(1,46)=64.80 ; p<0.001)$ : aftereffects with the right hand reached a similar magnitude in both groups, but only $29 \%$ of that magnitude with the unpractised left hand of group "single"; the practised left hand of group "dual" even showed slightly larger aftereffects than the right hand. Furthermore, ANOVA yielded significance for Modality $(\mathrm{F}(1,46)=15.39 ; p<0.001)$ : aftereffects with acoustic targets were only $66 \%$ of those with visual targets. No other interactions reached significance.

\section{Discussion}

The present study evaluates the transfer of sensorimotor adaptation to an unpractised limb and to an unpractised sensory modality, to scrutinize a recently published conceptual model (Bock, 2013). Our discussion will focus on the observed aftereffects rather than on the time-course of adaptation, since aftereffects are thought to be a pure indicator of sensorimotor recalibration while the time-course of adaptation may also reflect workaround strategies (McNay \& Willingham, 1998; Redding, 1996; Werner \& Bock, 2007).

The above model predicts that inputs from different sensory modalities are weighted equally, which implies that adaptation will fully transfer between modalities. This, however, was not the case in our study: following adaptation with visual targets, the magnitude of aftereffects with acoustic targets was only $66 \%$ of that with visual ones. The model therefore must be amended; specifically, if the practised visual modality in our study is given a weight of 1.00 , then the unpractised acoustic modality should obtain a weight of only 0.66 . Further work is 


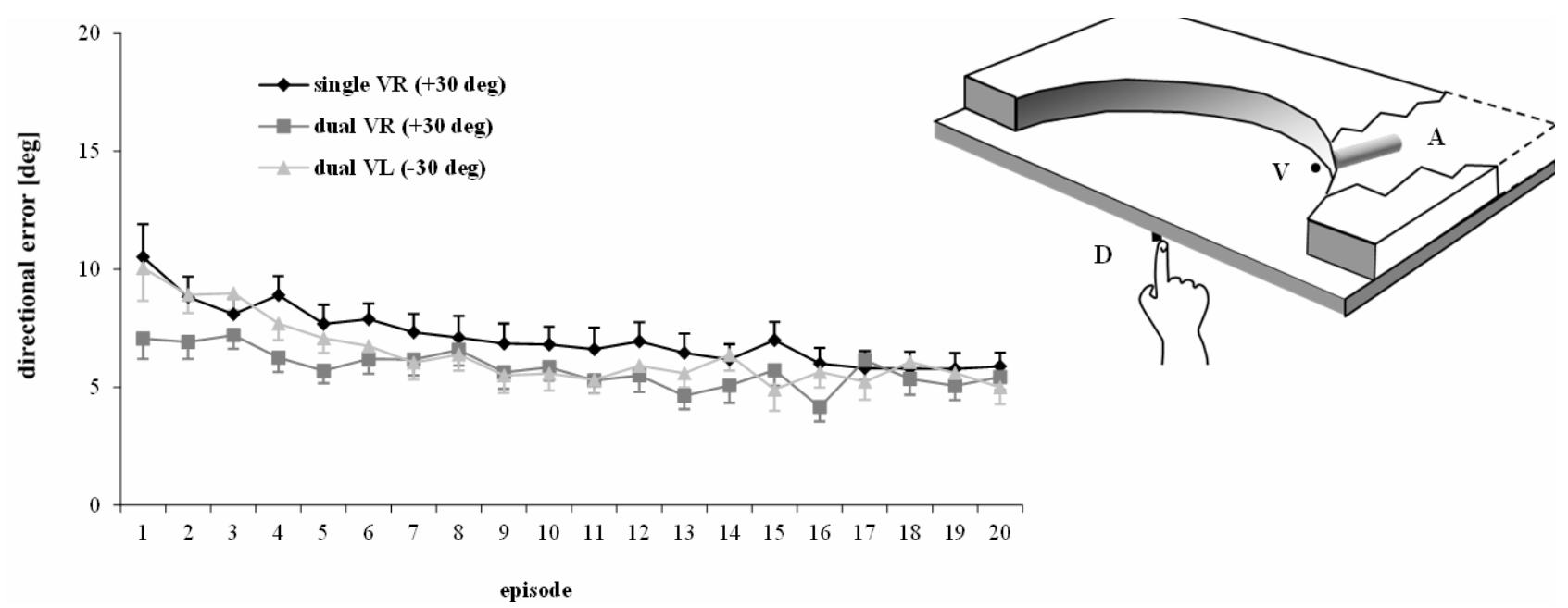

Figure 2.

Above-baseline errors during the adaptation block in groups "single" and "dual". VR and VL stand for visual rotation while using the right and left hand, respectively, with the type of rotation indicated in parentheses. Left-hand errors are inverted to facilitate comparisons. Symbols represent the across-subject means for each episode, and bars the corresponding standard errors. The inset is a sketch of the experimental setup: subjects pointed underneath an opaque panel, from a wooden dowel (D) towards visual (V) or acoustic targets (A) which were located along a semicircle around D; the hardware for delivery of acoustic stimuli was concealed from view, but one stimulus location is made visible in the sketch.

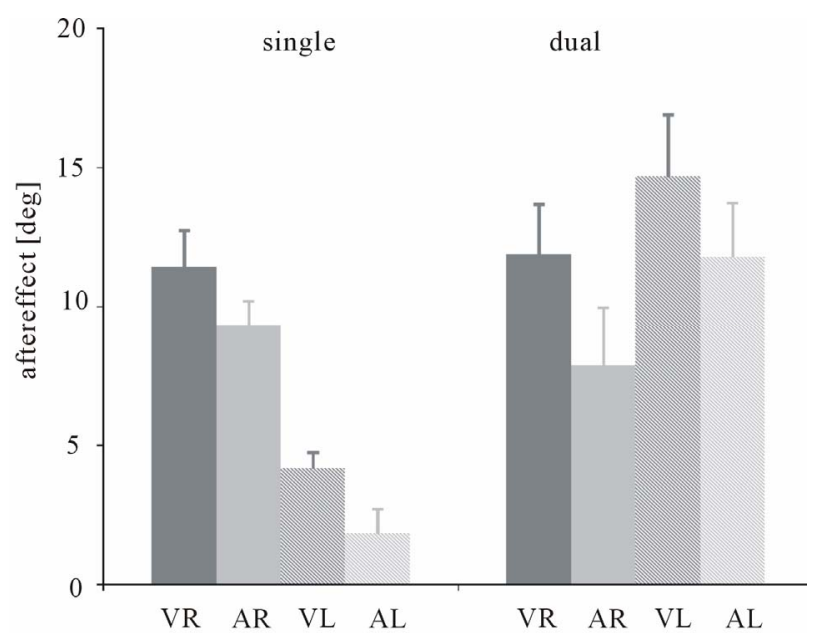

Figure 3.

Aftereffects in group "single" and "dual". Histograms represent acrosssubject means, and bars the corresponding standard errors. Left-hand data are inverted to facilitate comparisons. VR: visual targets and right hand, VL: visual targets and left hand, AR: acoustic targets and right hand, AL: acoustic targets and left hand.

needed to find out whether this lower weight is due to the lack of practise, or rather to the poorer localization of acoustic compared to visual targets.

Our finding, that input weights differ between sensory modalities, contrasts with the outcome of an earlier study, which found visual and acoustic aftereffects of similar magnitude following adaptation with visual targets (Kagerer \& ContrerasVidal, 2009). We suggest that this discrepancy is due to an artefact of fixed-order testing in the earlier study: acoustic aftereffects were always tested first and visual aftereffects second, such that the latter might already have declined by the time of testing.

The published model further predicts that there will be no interference between sensory modalities when both arms adapt to different distortions, which was indeed confirmed by our data: the difference between visual and acoustic aftereffects was comparable for both hands and groups. This might seem trivial at a first glance; note, however, that the left-hand data of group "dual" was sign-inversed for graphical presentation and statistical analysis. Without that transformation, it would be more obvious that visual and acoustic aftereffects of group "dual" were positive with the right hand, but both were negative with the left hand. Thus, differential adaptation of the right hand to +30 deg rotation and of the left hand to -30 deg rotation, achieved with visual targets, led to an unabridged differential transfer to acoustic targets.

As an additional outcome of the present study, we now can apply specific values to the output weights of the adaptation model. Given the different magnitude of aftereffects with the right and left hand of group "single", a weight of 1.00 for the practised right hand corresponds to a weight of 0.29 for the unpractised left hand. However, this outcome can't be generalized across all adaptation paradigms since different earlier studies yielded substantially different magnitudes of intermanual transfer, depending e.g. on the distribution of practise (Taub \& Goldberg, 1973) and on the provision of continuous versus terminal feedback (Cohen, 1967).

Summing up, the present study allowed us to confirm, modify and enumerate some aspects of a conceptual model of sensorimotor adaptation. We plan to scrutinize other aspects in future work.

\section{Acknowledgements}

This study was supported by the German Research Council DFG (Bo 649/8) and by the German Federal Ministry for Research and Technology (50WB9942). Responsibility for the contents rests with the authors.

\section{REFERENCES}

Alahyane, N. (2004). Eye position specificity of saccadic adaptation. 
Investigative Ophthalmology \& Visual Science, 45, 123-130. http://dx.doi.org/10.1167/iovs.03-0570

Bock, O. (1992). Adaptation of aimed arm movements to sensorimotor discordance: Evidence for direction-independent gain control. Behavioural Brain Research, 51, 41-50. http://dx.doi.org/10.1016/S0166-4328(05)80310-9

Bock, O., Worringham, C., \& Thomas, M. (2005). Concurrent adaptations of left and right arms to opposite visual distortions. Experimental Brain Research, 162, 513-519. $\mathrm{http}: / / \mathrm{dx}$.doi.org/10.1007/s00221-005-2222-0

Bock, O. (2013). Basic principles of sensorimotor adaptation to different distortions with different effectors and movement types: A review and synthesis of behavioral findings. Frontiers in Human Neuroscience, 7,81 .

http://dx.doi.org/10.3389/fnhum.2013.00081

Cohen, M. M. (1967). Continuous versus terminal visual feedback in prism aftereffects. Perceptual and Motor Skills, 24, 1295-1302. http://dx.doi.org/10.2466/pms.1967.24.3c.1295

Cohen, M. M. (1973). Visual feedback, distribution of practice, and intermanual transfer of prism aftereffects. Perceptual and Motor Skills, 37, 599-609.

http://dx.doi.org/10.2466/pms.1973.37.2.599

Cotti, J., Guillaume, A., Alahyane, N., Pélisson, D., \& Vercher, J.-L. (2007). Adaptation of voluntary saccades, but not of reactive saccades, transfers to hand pointing movements. Journal of Neurophysiology, 98, 602-612.

http://dx.doi.org/10.1152/jn.00293.2007

Kagerer, F. A., \& Contreras-Vidal, J. L. (2009). Adaptation of sound localization induced by rotated visual feedback in reaching movements. Experimental Brain Research, 193, 315-321. http://dx.doi.org/10.1007/s00221-008-1630-3

Malfait, N., Gribble, P. L., \& Ostry, D. J. (2005). Generalization of motor learning based on multiple field exposures and local adaptation. Journal of Neurophysiology, 93, 3327-3338.

http://dx.doi.org/10.1152/jn.00883.2004

McNay, E. C., \& Willingham, D. B. (1998). Deficit in learning of a motor skill requiring strategy, but not of perceptualmotor recalibration, with aging. Learning \& Memory, 4, 411-420. http://dx.doi.org/10.1101/lm.4.5.411

Prablanc, C., Tzavaras, A., \& Jeannerod, M. (1975). Adaptation of the two arms to opposite prism displacements. Quarterly Journal of Experimental Psychology, 27, 667-671.

http://dx.doi.org/10.1080/14640747508400527

Redding, G. (1996). Adaptive spatial alignment and strategic perceptual-motor control. Journal of Experimental Psychology: Human Perception and Performance, 22, 379-394. http://dx.doi.org/10.1037/0096-1523.22.2.379

Taub, E., \& Goldberg, I. A. (1973). Prism adaptation: Control of intermanual transfer by distribution of practice. Science, 180, 755-757. http://dx.doi.org/10.1126/science.180.4087.755

Thomas, M., \& Bock, O. (2010). Is sensorimotor adaptation to positionand velocity-dependent visual distortions based on distinct adaptive processes? Human Movement Science, 29, 179-186. http://dx.doi.org/10.1016/j.humov.2010.02.002

Thomas, M., \& Bock, O. (2012). Concurrent adaptation to four different visual rotations. Experimental Brain Research, 221, 85-91. http://dx.doi.org/10.1007/s00221-012-3150-4

Wada, Y., Kawabata, Y., Kotosaka, S., Yamamoto, K., Kitazawa, S., \& Kawato, M. (2003). Acquisition and contextual switching of multiple internal models for different viscous force fields. Neuroscience Research, 46, 319-331.

http://dx.doi.org/10.1016/S0168-0102(03)00094-4

Werner, S., \& Bock, O. (2007). Effects of variable practice and declarative knowledge on sensorimotor adaptation to rotated visual feedback. Experimental Brain Research, 178, 554-559. http://dx.doi.org/10.1007/s00221-007-0925-0

Woolley, D., Tresilian, J., Carson, R., \& Rick, S. (2007). Dual adaptation to two opposing visuomotor rotations when each is associated with different regions of workspace. Experimental Brain Research, 179, 155-165.

http://dx.doi.org/10.1007/s00221-006-0778-y

Zwiers, M. P., van Opstal, A. J., \& Paige, G. D. (2003). Plasticity in human sound localization induced by compressed spatial vision. $\mathrm{Na}-$ ture Neuroscience, 6, 175-181.

http://dx.doi.org/10.1038/nn999. 